\title{
Ventriculo-Peritoneal Shunt Infection Secondary to Translocation of Gut Bacteria without Evidence of Peritonitis
}

\author{
D Kang, Tarik Wasfie*, Avery Jackson, A Henke \\ Genesys Regional Medical Center, Genesys Trauma Services, Grand Blanc, MI, USA \\ *Corresponding author: Tarik Wasfie, Genesys Regional Medical Center, One Genesys Parkway, Grand Blanc, MI, 48439, USA; Email: Tarik.Wasfie@ascension.org
}

Received: March 20, 2019; Accepted: March 27, 2019; Published: April 20, 2019;

\begin{abstract}
Translocation of gut flora is a well-known and documented phenomenon which usually presents in immunocompromised patients or obstructive jaundice. Presented here is a patient with translocation of intestinal bacteria and subsequent infection of ventriculo-peritoneal (VP) shunt without clinical picture of acute peritonitis or sepsis.
\end{abstract}

Keyword: Ventriculo-peritoneal shunt, Bacterial translocation

\section{Introduction}

Translocation of gut flora resulting in sepsis in patients is well documented. However, translocation without a clear clinical picture of offending factors (i.e. immunosuppression, bowel obstruction, obstructive jaundice) are not well published. We present a patient who had a VP shunt with subsequent shunt infection by intestinal flora following enteritis secondary to Clostridium difficile bacteria.

\section{Case History}

Our patient is a 53-year-old female with medical history of cerebral aneurysm that was operatively clipped then subsequently developed hydrocephalus. The patient underwent placement of a VP shunt for the treatment of her symptomatic hydrocephalus. Patient presented to the emergency department 3 months later with a headache lasting 48 hours with photophobia, nausea, vomiting, and neck stiffness. Patient also reported two weeks of diarrhea. The patient denied any other significant findings on review of systems.

On physical exam the patient's vital signs were stable, afebrile, in no acute distress, lungs clear to auscultation bilaterally, and abdomen soft nontender nondistended. Laboratory findings upon arrival were a white blood cell count to $7.4\left(4.5-11.010^{3} / \mu \mathrm{L}\right)$, hemoglobin 15.2 $(11.0-17.3 \mathrm{~g} / \mathrm{dL})$, hematocrit 45.9 (36-53\%), platelet count 144 (140$\left.44010^{3} / \mu \mathrm{L}\right)$, sodium $141(136-144 \mathrm{mmol} / \mathrm{L})$, potassium $4.2(3.6-5.1$ $\mathrm{mmol} / \mathrm{L})$, chloride $103(101-111 \mathrm{mmol} / \mathrm{L})$, blood urea nitrogen 6 (8-26 mg/dL), creatinine $0.64(0.61-1.24 \mathrm{mg} / \mathrm{dL})$, glucose 108 (79-99 $\mathrm{mg} / \mathrm{dL})$, and anion gap of $16(3-13 \mathrm{mmol} / \mathrm{L})$. Throughout the entirety of the patient's hospital stay, she did not have an elevated white blood cell count, fever, tachycardia or tachypnea. She did however have asymptomatic bradycardia which was improved after discontinuing her beta-blocker medication.
CT of the brain showed proper position of ventriculostomy catheter placement and unchanged compared to previous CTs. There is no evidence of hemorrhage, midline shift, or hydrocephalus. CT of the abdomen was unremarkable with no acute abdominal or pelvic process visualized. Cerebrospinal fluid cultures were taken which were positive for enterococcus faecalis and E. coli. Clostridium difficile screen was positive for both antigen and active toxin in stool but blood cultures were negative. Patient was started on IV gentamicin, IV ampicillin, and oral vancomycin. Patient underwent successful removal of VP two-piece shunt and diagnostic laparoscopy with peritoneal washout. There was no evidence of free fluid or intraperitoneal infection. A moderate amount of adhesions were present within the abdominal cavity from previous surgeries but no abscess or phlegmon were found and no bowel perforation. Copious irrigation was performed with saline and sent for cultures which were negative for organisms and white blood cells. The patient tolerated the procedure well. She was ultimately discharged with IV gentamicin \& ampicillin, and oral vancomycin. Patient followed up 6 months later with improvement in her hydrocephalus without evidence of residual infection.

\section{Discussion}

VP shunt is the most common neurosurgical procedure done in the US [1]. This procedure often is complicated by infection resulting in the shunt being extracted, repositioned or replaced. While gut bacterial translocation is a common phenomenon [2,3], the pathophysiological outcome of such phenomena precipitating in VP shunt infection was not observed before.

The blood flow translocation presented by low immune response and intestinal infection has been observed in patients with acute abdominal pain, fever, nausea, and vomiting with signs and symptoms 
of peritonitis [4]. The management specifically directed toward the infection may be successful at time to salvage the shunt. Occasionally, temporary externalization of the distal portion of the shunt may be performed until the infection is controlled.

Bacterial translocation presented by the brief incidence of colitis is not well recorded. This brings the attention of the physician to the fact that disturbance of the gut flora and loss of intestinal mucosal barrier can occur with little or no symptoms of peritonitis and can potentially result in translocation of the gut bacteria and shunt infection. The plan for patient is immediate and aggressive treatment of the source of and removal of the shunt. Our patient had a brief history of diarrhea with few abdominal complaints and no signs or symptoms of peritonitis to explain the subsequent shunt infection. Furthermore, culture of peritoneal fluid was negative for bacteria. Therefore, the translocation of gut flora during the episode of colitis resulted in a loss of intestinal barrier integrity with subsequent positive CSF culture and VP shunt infection.

The conservative treatment with IV antibiotics at this stage was unsuccessful and ultimately to control the infection the shunt had to be removed. The long-term therapy in such patients is to continue IV antibiotics, followed by replacement of the shunt when needed.

We did not perform bacterial DNA-strain of the cultured organism due to the lack of resources, which would be helpful when available in similar future cases.

\section{Conclusion}

Intestinal bacterial translocation and its impact on the integrity of the VP shunt come with serious consequences. We presented a patient with very minimal evidence of intestinal infection and VP shunt infection, with failure of conservative management and ultimately removal of the shunt to achieve recovery.

\section{Acknowledgements}

The authors acknowledge Natalia Cwalina MD, for her invaluable assistance.

Declaration of Conflicting Interests: The Authors declare that there is no conflict of interest.

\section{References}

1. C Vaishnavi. (2013) Translocation of gut flora and its role in sepsis. Indian Journal of Medical Microbiology 31: 334-342. [Crossref]

2. S Balzan, C de Almeida Quadros, R de Cleva, et al. (2007) Bacterial translocation: overview of mechanisms and clinical impact. Journal of Gastroenterology and Hepatology 22: 464-471. [Crossref]

3. JC Dalfino, MA Adamo, RH Gandhi, et al. (2012) Conservative management of ventriculoperitoneal shunts in the setting of abdominal and pelvic infections. Journal of Neurosurgery Pediatrics 9: 69-72. [Crossref]

4. Y Gutierrez-Murgas, JN Snowden. (2014) Ventricular shunt infections: immunopathogenesis and clinical management. 276: 1-8. [Crossref]

\section{Citation:}

D Kang, Tarik Wasfie, Avery Jackson, A Henke (2019) Ventriculo-Peritoneal Shunt Infection Secondary to Translocation of Gut Bacteria without Evidence of Peritonitis. J Clin Res Med Volume 2(1): 1-2. 